Universidad de Panamá

Instituto de Estudios Nacionales
ISNN 1810-5491

Cuadernos Nacionales

$\mathrm{N}^{\circ} 26,99-111$, julio-diciembre, 2020

\title{
Contextualización Familia y Adolescentes: en riesgo o en conflicto con la ley ${ }^{36}$
}

\author{
Marisol Nicholsonl ${ }^{37}$ \\ marisolnicholson28@gmail.com
}

\section{Resumen}

En medio de una vorágine de cambios y transformaciones profundas desde el sistema económico, político, social y tecnológico, contextualizar la situación de la familia y los adolescentes en riesgo o en conflicto con la ley, por medio de datos e informes, publicados de la Política Pública de Juventud, la Situación de la Niñez y Adolescencia de Panamá y el Sistema Nacional Integrado de Estadísticas Criminales SIEC; buscamos elementos para conocer si los adolescentes están en riesgo o en conflicto con la ley y que factor cumple su familia (riesgo o de protección). Las divergencias en las normas legales para los adolescentes, sumada a la ausencia de investigaciones, obstaculizan el avance necesario para lograr estrategias y programas de prevención efectivos en la materia. Manteniendo a la familia y a los adolescentes enfrentados a múltiples estigmas y estereotipos, que han impactado en la opinión pública, incitando al aumento de las sanciones sin fundamento científico.

Palabras claves: Familia, adolescentes, Conflicto con la ley, riesgo, violencia.

\begin{abstract}
In the midst of a whirlwind of profound changes and transformations from the economic, political, social and technological system, contextualize the situation of the family and adolescents at risk or in conflict with the law, through data and reports published from the Policy Public Ministry of Youth, the Situation of Children and Adolescents of Panama and the Integrated National System of Criminal Statistics SIEC; We look for elements to know if adolescents are at risk or in conflict with the law and what factor their family meets (risk or protection).The divergences in the legal norms for adolescents, added to the absence of research, hinder the progress necessary to achieve effective prevention strategies and programs in this area. Keeping the family and adolescents facing multiple stigmas and stereotypes, which have impacted public opinion, inciting the increase of sanctions without a scientific basis.
\end{abstract}

Keywords: Family, adolescents, juvenile delinquency, risk, violence.

36. Recibido 22/6/2020 - Aprobado 15/7/2020

37. Licenciada en Trabajo Social. Estudiante de la Maestría en Ciencias Sociales con Especialización en Método y Técnica de Investigación Social, Universidad de Panamá. 


\section{Introducción}

Siempre escuchamos que el primer y más decisivo contexto evolutivo y educativo es el familiar, el cual debe jugar un rol protector de la niñez y la adolescencia. No obstante, es también en la familia en donde se presenta gran parte de los abusos y violencias contra ellos. El último informe (UNICEF, 2019) presentó que durante el año 2018 en Panamá, de las 9,945 personas menores de 18 años de edad ingresadas en los Juzgados de Niñez y Adolescencia por casos de protección, el $70 \%$ fueron atendidas por situaciones de abandono, descuido o negligencia por parte de los progenitores, el $17 \%$ por maltrato y un $13 \%$ por abuso sexual. En cuanto a los adolescentes en conflicto con la ley, solo reporto los pertenecientes al Instituto de Estudios Interdisciplinarios, que, al 30 de enero de 2019, mantenía una población total de 1269, de los cuales 408 se encuentran en centros de cumplimiento y centros de custodia, 182 se encuentran en centros penitenciarios de adultos y 679 se encuentran en algunas de las medidas no privativas de libertad. Una observación importante en estos datos fue la mención en los casos atendidos por el Juzgado de Niñez y Adolescencia, que aproximadamente la mitad de ellos, son niños y adolescentes que pertenecen a las provincias de Panamá y Panamá Oeste.

Mientras que el Sistema Nacional Integrado de Estadísticas Criminales, SIEC en sus boletines informativos de octubre 2019 y enero 2020 presentó un análisis de Panamá Oeste y San Miguelito, como las áreas mayormente afligidas por la violencia doméstica y con una alta tasa de delitos relacionados con problemas sociales. Además, presentaron las características en cuanto al índice de pobreza, mal servicio del transporte, debilidades en el sector salud, educación y problemas con los servicios públicos, donde ambas mantienen un mismo perfil. Tal situación es presentada solo como datos, y no como resultados de estudios que pudieran revelar factores puntuales, toda vez que el tema de la violencia interpersonal es reconocido como un factor de riesgo para la salud a lo largo de toda la vida y para los problemas sociales, según (Organización Mundial de la Salud, 2014).

Resultados de trabajos empíricos, realizados en la región revelan lo que transita la familia y adolescentes en una sociedad, sin trabajo, redes de contención, habilitaciones educativas y sociales exigidas por el mercado, ni oportunidades para obtenerlas, quedando fuera de la sociedad formal y se refugian en las estructuras invisibles de la pobreza y la marginalidad (Jimenez Omelas, 2005). Como también otros comparan, la distribución de variables familiares y sociales entre adolescentes en conflicto con la ley penal y adolescentes escolarizados de la 
población general desde variables como: conflictos familiares, interacciones afectivas (apego), comunicación, supervisión, entre otras, revelando similitudes y diferencias entre adolescentes que han estado o están en conflicto con la ley y los que no.

En nuestro país, no se ha evidenciado estudios que aborden las relaciones familia como factor de protección o de riesgo en los adolescentes. Sin embargo, al ver los pocos datos aislados que encontramos, aflora la necesidad de realizar estudios que nos permitan acercarnos a una realidad que enfrenta las familias con hijos adolescentes, en las áreas reconocidas como las más afligidas por la violencia y delitos en nuestro país.

Al considerar los delitos automáticamente tenemos que hacer un recuento en nuestras normativas dirigidas a las personas menores de edad, sabiendo que estas han evolucionado de una manera muy particular, al punto que hoy tenemos una ley que clasifica dos grupos etarios, dentro de las personas menores de edad; donde el primer grupo etario está totalmente invisibilizado en todos los datos que han presentado los informes de alto perfil que utilizamos para esta contextualización. conduciéndonos a la búsqueda de los factores que giran entre la familia y la adolescencia en riesgo y/o delito.

\section{Familia}

Hablar de familia en la actualidad implica, necesariamente, tener en cuenta una amplia tipología que va desde la familia clásicamente entendida: padre, madre e hijos, hasta otros tipos cada vez más extendidos, como padres y madres solteros, separados, divorciados, familias donde conviven hijos de distintos matrimonios, parejas de un mismo sexo, adolescentes que identifican a sus padres sin que estos formen parte de su grupo familiar, clasificándolas en otros tipo de familia, por no responder a los tradicionalmente existentes.

Los documentos legales y normativos de nuestro país son los que nos aproximan a la definición del concepto de familia, es decir el ámbito jurídico, al integrar varios artículos de la Ley No. 3 de 17 de mayo de 1994 que aprobó el Código de la Familia. Toda vez, que la ausencia dentro de las leyes que han creado y/o modificado las Instituciones que atienden directamente a la familia y a los adolescentes, no la definen, aceptando un modelo tradicional, que sin dudas ha evolucionado. Por ello, considerando una definición no tradicional del concepto familia que responda a la descripción de la amplia tipología antes mencionada, la encontramos en (Balbi \& Boggiani, 2015) quienes la definen como un sistema de relaciones sobre todo afectivas, en el que el ser humano permanece largo tiempo y que acompaña al individuo en sus fases evolutivas 
fundamentales, el ambiente social en el que las mentes adultas _padres o tutores-interactúan con individuos que se están formando, o sea, los hijos.

Hace 30 años, organizaciones como CEPAL - UNICEF y Las Naciones Unidas, iniciaron estudios en la región sobre la evolución de las estructuras familiares en el periodo de 1990-2010, describiendo los cambios complejos que las familias están experimentando, como: el aumento sostenido de los hogares unipersonales, falta una conexión, el descenso significativo de los hogares nucleares biparentales, la marcada tendencia de aumento de los hogares monoparentales con jefatura femenina, como también los extensos; recalcando la necesidad de estudiarlos con más profundidad. (Ullman \& Nieves, 2014).

\section{Normativas}

Durante la vida republicana del país, hemos tenido diversos cambios en el sistema de justicia, por ello, un breve recorrido lo iniciamos con el Código Civil, Judicial y Penal, y en el Código Administrativo de (1916 y 1917), que establecieron que las personas menores de edad no tenían derecho a la defensa, pues no se menciona. Sin embargo, si eran objetos de castigo y represión, desde los 7 años, contemplaban como la edad mínima para ser arrestados. En 1922 se reformó, aumentando a 12 años la edad mínima de arresto, manteniéndose el derecho a nombrar defensor de juicio penal solo al imputado mayor de edad. Estas leyes prevalecieron casi 30 años, y fue en 1951 cuando se crea por medio de la Ley 24, el Tribunal Tutelar de Menores, dejando atrás el modelo penal. El modelo tutelar tiene la característica fundamental que los adolescentes, o en general los niños y niñas, son considerados como un objeto de protección y no como sujetos de derecho, lo que significaba que el sistema de justicia decide por el adolescente, sin escucharlos; considerando con esto que no se les podía atribuir ninguna responsabilidad penal. De ahí que la comisión de un hecho de delito, tenga la particularidad de formar parte de las diferentes conductas, en la que se enmarca la situación irregular en la que se encuentran estos sujetos; pero no solo entraba en esta la comisión del delito, sino que abarcaba las circunstancias difíciles como abandono y negligencia (Tiffer \& Deniel, 2012).

En 1990, cuando se firmó y ratificó la Convención sobre los Derechos del Niño, junto a dos importantes resoluciones relacionadas con el fenómeno de la delincuencia juvenil como lo son: Las Directrices para la prevención de la delincuencia juvenil (Resolución 45/112) y Reglas para la protección de menores privados de libertad (Resolución 45/113). Sin embargo, la 
convención no hace mención al termino adolescente, entendiendo por niños a todo ser humano menor de dieciocho años de edad.

Estas normas internacionales iniciaron un proceso de transformaciones en nuestro sistema de justicia juvenil, buscando garantizar el bienestar basado en derechos, de todas las personas menores de 18 años, marcando una pauta fundamental de evolución del "Derecho Tutelar" que prevaleció aproximadamente medio siglo, hasta que en 1999 llegamos a la responsabilidad del adolescente, por medio de la Ley 40 de 26 de agosto de 1999, del Régimen Especial de Responsabilidad Penal para la Adolescencia, en su última modificación en el año 2010, clasificó dos grupos etarios: 1. A partir de los doce años de edad y hasta que no hayan cumplido los quince años de edad. 2. A partir de los quince años de edad y hasta que no hayan cumplido los dieciocho años de edad. Además de plasmar una distinción Institucional, por edades al mencionar que el primer grupo etario está bajo la supervisión de la Secretaria Nacional de Niñez, Adolescencia y Familia SENNIAF, por ende, el segundo es atendido por el Instituto de Estudio Interdisciplinario.

Elemento que deja una duda sustancial entre la ley 40 de 1999, cuando es especifica de la responsabilidad penal para la adolescencia, mientras que la ley 14 del 23 de enero de 2009, que crea la SENNIAF establece como niño o niña a toda persona desde su concepción hasta antes de cumplir los 14 años y adolescentes a toda persona que habiendo cumplido los 14 años de edad no ha cumplido los 18 años. Los intervalos de tiempo, en las modificaciones de los sistemas de justicia nos indican sin duda, que nos acercamos a nuevas transformaciones que aún no han sido reveladas, sumado a la casi resiente implementación del Sistema Penal Acusatorio, que en algún momento este deberá incluir al sistema de justicia juvenil.

\section{Jóvenes}

Desde las normativas apreciamos que existen discrepancias en los rangos de edades que nos lleven a determinar concretamente por medio de ellas la adolescencia; de manera el termino juventud, aunque más abarcador encierra de cierta manera la adolescencia, al considerar dentro de las políticas públicas a los jóvenes entre las edades de 15 a 19 años. En 1985, se celebró el primer Año Internacional de la Juventud (AIJ), con la contribución de organizaciones no gubernamentales como la CEPAL, algunas Agencias de las Naciones Unidas y la Cooperación Española, se comenzaron a realizar los primeros estudios serios sobre la juventud en la región y a construirse cierta institucionalidad pública en relación con estas temáticas en los primeros 
documentos de "Política Nacional de Juventud", conteniendo dentro de esta la población adolescente. Por ello, las corrientes o enfoques políticos de cada país deben formar parte de los análisis en esta temática, en recientes compilaciones de estudios en la región encontramos quienes advierten de procesos complejos:

en los que se disputan enfoques, estrategias e intereses muy marcados, en cuyo marco, las y los jóvenes son vistos como un simple grupo de riesgo al igual que visibiliza a la población como "consumidores" más que como "ciudadanos" (en el enfoque neo-liberal y neo-conservador); mientras el otro enfoque los visualiza como sujetos de derecho, asumiendo la pertinencia de prevenir violencias y "empoderar" a la ciudadanía para su participación activa en el desarrollo (en el enfoque neo-desarrollista)" (Vásquez, Ospina-Alvaro, \& Domínguez, 2018).

A pesar de los avances alcanzados en materia de juventud, también se nos advierte que estas "tensiones" entre enfoques han negado la posibilidad de implementar en la práctica, las transformaciones recomendadas por las normas internacionales, sin mencionar la debilidad institucional que tenemos para hacer efectivas la real implementación de todo lo recomendado. "En este marco, las juventudes se encuentran implicadas en disputas muy diversas, discriminadas y estigmatizadas por los discursos dominantes, sin que sus principales problemas sean encarados seriamente y sin que sus reclamos de participación encuentren -salvo excepciones-el eco correspondiente" (Vásquez, Ospina-Alvaro, \& Domínguez, 2018).

\section{Antecedentes}

Tras una revisión bibliográfica en las bases EBSCO, e-libro, Google académico y Biblioteca Interamericana Simón Bolívar no se han localizado estudios locales publicados que aborden las relaciones o diferencias entre dinámica familiar y los menores en conflicto con la ley y los que no han infringido la ley (de la población general).

Mientras que desde las ciencias sociales son diversas las investigaciones relacionadas a comprender las estructuras y dinámicas de la familia y los adolescentes en conflicto con la ley, y los que no, evidenciando que, esta problemática ha sido analizada desde diferentes posicionamientos teóricos. Para el caso de América Latina, las investigaciones en su gran mayoría se han orientado en comprender la influencia de la familia en el aprendizaje de las conductas delictivas de los adolescentes. Lo anterior es evidenciado en los estudios de Redondo, Fernández y López (1988); Valgañon, M., Muñoz, L., Ferrandiz, A y Quiroga, M. P. (2016); Tenenbaum (2016); Cardona, E., Martínez, N., Klimenko, O. (2017). 
Muchos de ellos por medio de las variables afectivas tales como: apego, cohesión y conflicto familiar, así como la supervisión y el control de los padres. Sus principales conclusiones dan cuenta que, los adolescentes delincuentes se diferencian de los no delincuentes, en cuanto a las relaciones afectivas y a los aspectos normativos de su entorno familiar. Logrando verificar que,

“...El apego se revela como la variable fundamental para establecer diferencias entre los delincuentes y los no delincuentes. Los sujetos no delincuentes experimentan un grado de apego hacia sus padres significativamente mayor que los delincuentes." (Redondo, Fernández y López, 1988.p177)

Por su parte, en el análisis de las dimensiones cohesión y conflicto familiar, la investigación deja clara evidencia estadística de que “...las familias de los adolescentes delincuentes se caracterizan por un bajo nivel de cohesión y un alto nivel de conflicto" (Redondo, Fernández y López, 1988.p178). Continuando con las investigaciones empíricas más reciente, que igualmente resaltan la importancia de los vínculos afectivos con personas socialmente integradas, como mecanismo primario que retiene a los jóvenes de implicarse en actividades delictivas (Bobbio, Lorenzino, \& Arbach, 2016). También hay un estudio comparativo entre niños de Argentina y España, donde se valoraron estilos parentales, inestabilidad emocional y agresividad, encontrando que los comportamientos parentales negligentes, en especial los de la madre, son el predictor más importante de inestabilidad emocional y agresividad en los niños, y que estos resultados permanecen invariantes a través de los estratos socioeconómicos y de las poblaciones estudiadas (Richaud, y otros, 2013).

Algunos explican cómo el contexto familiar hace posible el proceso de orillamiento de los adolescentes a tener una mayor exposición al riesgo de cometer una infracción, por medio de entrevista a profundidad, revelando que las madres desenvolviendo su rol solas, o al lado de un progenitor varón que es periférico o aglutinado, se observa persistencia en la distancia afectiva, desligamiento relacional, apego distante, resonancia escasa se puede pensar en que la pauta organizacional es la evitación y el joven infractor no encuentra en su familia posibilidades de apoyo o acompañamiento funcional a sus demandas y temores. (Tenenbaum, 2016). Otros revelaron que, en la mayoría de las familias, la figura paterna se encuentra ausente física o simbólicamente, promoviendo una mayor vinculación al referente materno. Destacó una comunicación inadecuada e ineficaz no permite generar una buena cohesión familiar, por ende, tampoco se establecen unas rutinas dentro de las actividades cotidianas, limitándose solo al hecho de compartir un espacio físico de la casa, generando en los jóvenes percepciones de soledad (Cardona, Martínez, \& Klimenko, 2017). 
Trabajos, enfocado en las transformaciones sociales y demográficas de las familias latinoamericanas, plantearon que se han construido estructuras familiares a partir de la información de parentesco en relación con el jefe del hogar. Concluyendo que estos cambios sociales, económicos y culturales han incidido en las relaciones internas de las familias, en las mentalidades y las prácticas sociales (Arriagada, 2004). Situación que no escapa a nuestra realidad, ya que la escaza literatura en los cambios de las estructuras familiares, especialmente en el ámbito jurídico, nos indica el posible atraso que tenemos respecto a la realidad y la falta de actualización de los programas existentes en atención de la materia.

Algunos contextualizaron con el perfil socio-económico de los adolescentes que han presentado delincuencia juvenil: como un fenómeno de la sociedad actual, realizada por medio de entrevistas de percepciones revelando que (sin escolaridad, primaria, secundaria, bachillerato y estudios superiores) y el análisis de la situación económica y política de México, señalo en sus conclusiones que sin trabajo, sin redes de contención, sin las habilitaciones educativas y sociales exigidas por el mercado ni oportunidades para obtenerlas, estos jóvenes quedan fuera de la sociedad formal y se refugian en las estructuras invisibles de la pobreza y la marginalidad. Dirigiendo la mirada a que los jóvenes se encuentran ante las actividades ilegales que ofrece la marginalidad urbana, como única posibilidad de movilidad social (Jimenez Omelas, 2005).

Otros estudios han realizado contrastes entre adolescentes en conflicto con la ley penal y con adolescentes que estudian normalmente sin haber cometido delito, como el de Menores infractores: un estudio de campo de los factores de riesgo de tipo psicológico-individual, como: autoconcepto, socialización, afrontamiento e inteligencia emocional, encontrando que no detectaron diferencias en autoconcepto entre menores infractores y el del grupo normativo en ninguna de sus principales dimensiones (académica, social, emocional y familiar). Sin embargo, los infractores obtuvieron niveles inferiores de inteligencia emocional en contraste con los normativos. Como también los menores infractores poseen niveles inferiores de socialización, afrontamiento e inteligencia emocional, reflejando menos competencia y destrezas sociales, que aumenta su vulnerabilidad. (Vásquez, Amado, \& Alves, 2013).

Los trabajos mencionados sobre el tema familia y adolescentes, revelan convergencias en que las relaciones y comunicaciones familiares, demarcan diferencias entre los adolescentes en conflicto con la ley y los que no están en conflicto con la ley o no delincuentes; cuyos estilos parentales pueden aumentar el comportamiento delictivo o no. Al igual que han revelado algunos instrumentos que van desde pruebas psicométricas que se han adaptado a la cultura de un país, 
como también cuestionarios validados para la región, que sin dudas se vuelven fundamentales en futuros estudios, con parámetros comparativos a nivel de región.

\section{Recorrido teórico}

A nivel teórico son muchos son los autores que contribuyen al análisis del tema, aun cuando estos pertenecen a distintas corrientes sociológicas. Iniciando con Emile Durkheim, planteo hace más de cien años que toda educación consiste en un esfuerzo continuo por imponer al niño formas de ver, de sentir y de actuar a los cuales no llegaría espontáneamente, están dotados de un poder de coacción que presentan la propiedad notable de que existen fuera de las conciencias individuales. Donde los padres de familia y maestros no son más que representantes e intermediarios que replican la presión del medio social (Durkheim, 1986). Mientras, Lode Walgrave revela en sus análisis, que toda sociedad ofrece cierto número de bienes y servicios al ciudadano: la educación, la formación, la información, la asistencia, incluso apoyo material, la seguridad, y en algunos casos, hasta el prestigio y el confort, pero esa oferta no es gratuita, ella está condicionada; a cambio de ella, los ciudadanos deben someterse a las reglas de la sociedad, es ahí donde se encuentra el lado del control (Morales \& Castillo, 2008).

Al hablar de control, en 1975, Foucault, en su intento de comprender el poder desde su esencia, motivo por el cual analizo la multiplicidad de técnicas que aparecen neutras, describiendo con ello la microfísica del poder. El panóptico de Bentham, descrito por Foucault lo podemos aproximar a lo cotidiano de hoy; a las tendencias seguidas según los enfoques políticos, impuesta por la sociedad, en la cual las redes sociales y medios de comunicación juegan un papel fundamental. Mencionando este autor que las instituciones son en sí mismas dispositivos de disciplinamiento, por medio de la vigilancia en busca de una normalización generalizada. Mientras Bourdieu sostuvo "que las formas de clasificación son formas de dominación, que la sociología del conocimiento es inseparablemente una sociología del reconocimiento y del desconocimiento, es decir, de la dominación simbólica". La cual podemos hacerla mucho más operativa con el concepto de habitus, que la familia es la institución por excelencia donde se gesta una verdadera labor ritual y técnica orientada a instituir duraderamente a cada uno de sus miembros mediante ritos que producen conductas; que a su vez podemos encontrar diversos tipo de habitus, los cuales contribuyen a clasificar o distinguir diferentes clases sociales dentro de una misma sociedad (Manzo E. , 2010).

Siguiendo una secuencia, tenemos La construcción social de la realidad, como interacción, intersubjetividad y universo simbólico; de Luckmann en torno a la comunicación en 
la vida cotidiana describe como los procesos se naturalizan y luego se obvia que ha sido construido e instruido por y para un determinado fin. Centrando esta teoría en el sujeto que concibe, antes que cualquier otra cosa, como un individuo en permanente vínculo e interacción con sus semejantes; desde estas situaciones de interacción, los sujetos construyen la sociedad y, a la vez, son construidos por ésta. (Berger, Luckmann, \& Zuleta, 1968). Por lo antes mencionado el tema familia y adolescente, circula entre Durkeim, Bourdieu y Luckmann, los que dan sentido a nuestra búsqueda de elementos que se ven naturales o espontáneos, invisibilizado algunos actores y factores de incidencia directa, que muchas veces los medios mediáticos "bajo el discurso" desde la dominación del poder político, cultural y por ende económico, son los interesados en direccionar las acciones de la sociedad dividida en clases sociales. Recordando palabras de Gramsci, en el concepto de hegemonía como la capacidad de unificar y mantener unido a través de la ideología un bloque social que no es homogéneo.

\section{Reflexión}

Bajo estos enfoques podremos valorar cada detalle de las situaciones que giran alrededor de las familias y los adolescentes en riesgo y/o conflicto con la ley, bajo la constante vigilancia epistemológica para no dar por hechos o por dado situaciones que se manipulen desde afuera, sino encontrar realidades que están ante nuestra mirada y no son fáciles de distinguir por los procesos de naturalización en que estamos inmersos, anunciados por los teóricos mencionados. Son muchos los autores que han advertido la existencia de la crisis en la familia, haciendo referencia al debilitamiento de un modelo de familia basado en el ejercicio estable de la autoridad/dominación en el hombre adulto cabeza de familia. Todo esto trae consigo transformaciones y nuevas tendencias, anunciadas en los resultados de estudios que han incluido variables demográficas y socioculturales.

Los estudios mencionan en sus resultados que la comunicación inadecuada e ineficaz no permite generar una buena cohesión familiar, las familias en sus dinámicas no poseen rutinas dentro de las actividades cotidianas de convivencia, se orientan hacia la evolución regresiva, las madres presentaron sobre aumento de roles, que llevan a desdibujar límites; en cuanto a los padres muestran ausencia o periferia, teniendo como resultado una persistencia en la distancia afectiva, desligamiento relacional, apego distante, con evitación limitándose solo al hecho de compartir un espacio físico de la casa, que los lleva a crear fuera de la familia otro tipo de vínculos que buscan dar respuesta a su necesidad de reconocimiento, sin importar que tan adecuados sean estos, además que han diferenciado los adolescentes en conflicto con la ley y nos 
que no. Esta contextualización como medio de aproximación a la realidad concreta de una población enfrentada a múltiples estigmas y estereotipos, que han impactado en la opinión pública mostrando muchas veces a los adolescentes como "buenos para nada" y "culpables hasta que demuestren su inocencia (vigilar y castigar), incitando al aumento de las sanciones. El panorama ofrecido en (Vásquez, Ospina-Alvaro, \& Domínguez, 2018) refieren como se disputan enfoques, estrategias e intereses muy marcados, distantes de una igualdad de beneficios para esta población que escasamente le han brindado un tipo de vida digna en la sociedad, en la que ellos no toman, ni forman parte de las decisiones de su entorno social, a pesar de los documentos internacionales ratificados por el país que llevan años de haber sido propuestos. En una propuesta para diseñar estrategias de prevención adaptadas a las necesidades específicas de la población adolescente, seria de valor un estudio que contenga la distribución de variables familiares y sociales entre adolescentes en riesgo o en conflicto con la ley penal, de manera que pueda ser comparado con los resultados de otros estudios en la región; que han señalado que las estructuras familiares y sus dinámicas, pueden proteger o exponer un comportamiento en los adolescentes, considerando el contexto en que se desarrolla, es esencial para las Ciencias Sociales, de manera que sus resultados muestren la realidad objetiva, en medio de una vorágine de cambios y transformaciones profundas que tenemos desde el sistema económico, político, social y tecnológico.

Sumado a estos fundamentos encontramos en las consultas de las políticas públicas de juventud de Panamá, algunas voces de jóvenes que reconocieron sus preocupaciones sobre el ámbito familiar, tales como la falta de afecto, de dialogo y de tiempo en compañía de sus padres, la violencia intrafamiliar y la difícil situación económica que genera crisis familiares" (Ministerio de la Juventud, 2004). Reconociendo que la familia es el ente socializador por excelencia, sumado a la solicitud de reformas a nuestra constitución, el ingreso reciente al sistema penal acusatorio, una ley de responsabilidad penal para la adolescencia con más de 20 años de vigencia, la Estrategia de Seguridad Ciudadana 2017-2030, los informes del SIEC, entre otros documentos; consideramos la importancia que representa realizar el estudio "Estructuras Familiares y el comportamiento de los adolescentes en riesgo o en conflicto con la ley, en Panamá Oeste. Análisis desde las trayectorias de vida”. Que ilustre nuestra realidad panameña, haciendo posible una comparación con estudios de la región y base para la implementación de estrategias y programas de prevención en la materia.

\section{Referencias}


Arriagada, I. (2004). Transformaciones sociales y demográficas de las familias latinoamericanas. Papeles de población, 71-95.

Balbi, E., \& Boggiani, E. (2015). Adolescentes violentos: con los otros, con ellos mismos. Barcelona: Herder.

Berger, P., Luckmann, T., \& Zuleta, S. (1968). La construcción social de la realidad. Buenos Aires: Amorrorlu.

Bobbio, A., Lorenzino, L., \& Arbach, K. (2016). Familia, barrio y sociedad: un estudio comparativo en jóvenes con y sin antecedentes delictivos de Argentina. Revista Criminalidad, 81-95.

Durkheim, E. (1986). Las Reglas del Método Sociológico. México: Fondo cultural economía.

Jimenez Omelas, R. (2005). La delincuencia juvenil: fenómeno de la sociedad actual. Papeles de Población, 215-261.

Cardona, E., Martínez, N. M., \& Klimenko, O. (2017). Estudio sobre las dinámicas familiares de los adolescentes infractores del programa AIMAR del Municipio de Envigado, durante el año 2016. Revista Katharsis, 23.

Manzo, E. (2010). Las teorías sociológicas de Pierre Bourdieu y Norbert Elias: los conceptos de campo social y habitus. Estudios sociológicos, 383-409.

Ministerio de la Juventud, 1. M. (2004). Política Pública de Juventud de Panamá. Panamá.

Morales, J. \& Castillo. (2008). La vulnerabilidad societal, el rol de la familia, la teoría integrativa de Lode Walgrave para explicar la delincuencia juvenil. Justicia (Barranquilla), 71 - 78.

Organización Mundial de la Salud, O. (2014). Informe sobre la situación mundial de la prevención de la violencia. Suiza.

Redondo, L., Fernández, A., \& López, J. (1988). Un análisis de la relación entre ambiente familiar y delincuencia juveni. Revista de Psicología Social, 165-180.

Richaud, M., Mesurado, B., Samper-García, P., llorca, A., Lemos, V., \& Tur, A. (2013). Estilos parentales,inestabilidad emocional y agresividad en niños de nivel socioeconómico bajo en Argentina y España BAJO EN. Ansiedad y Estrés, 53-69.

Tenenbaum, G. (2016). Infracción y desistimiento: influencias familiares en los adolescentes en conflicto con la ley de la Ciudad de México. Revista mexicana de ciencias políticas y sociales, 195-221.

Tiffer, C., \& Deniel, A.-J. (2012). Justicia Penal Juvenil. Costa Rica: Instituto Lationoamericano de las Naciones Unidas.

Ullman, H., \& Nieves, C. M. (2014). La evolución de las estructuras familiares en América Latina, 1990 - 2010. CEPAL - UNICEF, 1-63.

UNICEF. (2019). Situación de la niñez y adolescencia en Panamá. Panamá: Fondo de las Naciones Unidad para la Infancia.

Valgañon, M., Muñoz, L., Ferrandiz, A., \& Quiroga, M. (2016). Familias de adolescentes infractores penales reiterantes: funcionamiento, roles y coaliciones parentales. 
Vásquez, M. V., Amado, B. G., \& Alves, C. (2013). Menores infractores: un estudio de campo de los factores de riesgo. Anuario de psicología jurídica, 39-45.

Vásquez, M., Ospina-Alvaro, M., \& Domínguez, M. (2018). JUVENTUDES E INFANCIAS en el Escenario Latinoamericano y Caribelo actual. Argentina: Consejo Latinoamericano de Ciencias Sociales CLACSO. 